\title{
Opiniones y propuestas de los estudiantes de Comunicación Audiovisual sobre los SPOC
}

\section{Opinions and proposals from Film, TV and Media students about SPOCs}

\author{
Ana Mejón \\ Francisco Utray \\ David Rodríguez-Mateos \\ Universidad Carlos III de Madrid (España)
}

\section{Resumen}

En este artículo se presentan los resultados de la investigación realizada con estudiantes universitarios del Grado en Comunicación Audiovisual sobre la implementación de cursos SPOC («Small Private Online Course») en las clases de contenido práctico. La motivación para realizar este trabajo surge de la disparidad identificada en las aulas del nivel de habilidad de los estudiantes para la operación de los dispositivos tecnológicos. Los objetivos de la investigación han sido conocer cuáles son para ellos los valores de utilidad de las herramientas de «blended learning» y qué competencias profesionales de producción audiovisual consideran susceptibles de desarrollarse mediante SPOC. La investigación se realizó de forma simultánea a la impartición de un curso piloto sobre edición de vídeo y el análisis se llevó a cabo mediante sesiones grupales de discusión y encuestas individuales. Como resultado se han obtenido una lista cualificada de valores de utilidad y unas propuestas de contenidos para complementar la docencia presencial. Los estudiantes han expresado de forma muy homogénea la conveniencia de disponer de materiales didácticos en línea sobre operación de los dispositivos tecnológicos mediante unos cursos que estén abiertos y accesibles para poder aprender a «su propio ritmo» y volver a ellos cuando sientan la necesidad de repasar a lo largo de toda la carrera.

Palabras clave: educación; enseñanza de los medios de comunicación; enseñanza a distancia; aprendizaje; medios audiovisuales; universidad.

\section{Abstract}

This article presents the results of the study carried out among a group of students from a B.A. in Film, Television and Media Studies, on how to implement a SPOC ( Small Private Online Course») as part of a practice course. Classroom experience has revealed a noticeable gap in the students' technical skills when operating the equipment. The aim of the research was to assess the utility values attributed by the students to blended learning tools and to find out which audiovisual production skills they find fit to be developed through a SPOC. The research was conducted simultaneously with a pilot SPOC on video editing and the assessment was elaborated through group discussions and individual polls. As a result, a preliminary list 
of SPOC utility values was drawn and content proposals to complement face-to-face learning were put forward. Students widely supported the view of having online material on technical devices operation available anytime throughout their degree course, so that they can learn at their own pace.

Keywords: education; media; blended learning; higher education.

En las clases de prácticas del Grado en Comunicación Audiovisual en la universidad, los estudiantes tienen que realizar ejercicios de fotografía, sonido y vídeo que implican el manejo de dispositivos tecnológicos complejos como las cámaras, los micrófonos o los programas informáticos para la postproducción. Tal como queda recogido en el Libro Blanco de los Estudios de Comunicación (ANECA, 2005, p. 325), los planes de estudio tienen que contemplar la formación en habilidades de operación de las herramientas de producción a través de contenidos instrumentales obligatorios, orientados a formar en el uso adecuado de herramientas tecnológicas en las diferentes fases del proceso audiovisual. El objetivo docente es desarrollar en los estudiantes una serie de competencias profesionales que les permitan adquirir habilidades relacionadas con la elaboración, composición, acabado y masterización de diferentes productos audiovisuales y multimedia (Universidad Carlos III de Madrid, 2017).

A pesar del esfuerzo de actualización de los planes de estudio realizado en los últimos años, la realidad es que no hay tiempo suficiente para cumplir este objetivo con solvencia y muchos estudiantes se encuentran limitados por falta de formación básica en este ámbito. Los profesores, por su parte, se tienen que enfrentar a una gran disparidad en los niveles de conocimientos y habilidades entre los estudiantes que forman cada grupo de docencia: algunos son expertos en tecnología y otros, sin embargo, se sienten incómodos en las tareas técnicas y con el manejo avanzado de las herramientas. Esta disparidad plantea un dilema para el profesor de prácticas: si el curso se dirige a los más avanzados, los estudiantes con menos experiencia quedan excluidos; si, por lo contrario, se orienta a un perfil más básico, se baja el nivel de la clase y los mejores estudiantes pierden la motivación y el interés.

En la búsqueda de vías para solucionar esta problemática, se ha experimentado la utilización de los modelos docentes de aprendizaje semi-presencial («blended learning») en asignaturas que hacen uso de los laboratorios audiovisuales (Utray, 2016).

Como hipótesis se ha considerado que las metodologías docentes que combinan el aprendizaje en línea («online») con las clases presenciales en el Grado en Comunicación Audiovisual pueden servir para enriquecer el modelo docente y favorecer la adquisición de las competencias básicas de operación de los dispositivos tecnológicos de la producción audiovisual.

Numerosos autores han tratado en profundidad las barreras y las oportunidades de modelo semi-presencial en la educación superior, tanto en aproximaciones que 
datan de más una década (Alexander, 1999; Nunan, George y McCausland, 2000; Voos, 2003; Lim y Kim, 2003; Graham, 2004; Bernard y otros, 2004; Harding, Kaczynski y Wood, 2005), como en contribuciones recientes (Porter y Graham, 2016). Desde la perspectiva crítica, se ha señalado que la promoción de cursos en línea puede responder más a intereses de gestión universitaria que a la mejora de la docencia. Y también, que aún está por determinar el modelo y la sostenibilidad de las iniciativas que se han puesto en marcha en estos últimos años (Bartolomé y Aiello 2006; Bartolomé y Steffens, 2015; Chiappe-Laverde, Hine y Martínez-Silva, 2015).

Las metodologías docentes de la semi-presencialidad giran en torno a la idea de la clase invertida («flipped classroom»), una reconfiguración del modelo de enseñanza basado en las clases magistrales del profesor. En la enseñanza tradicional, el formador utiliza el tiempo de clase para presentar nuevos conceptos e información en forma de ponencia documentada con ejemplos y demostraciones. Es lo que se conoce como docencia centrada en el profesor («teacher-centered approach»). El modelo de clase invertida es un formato más colaborativo basado en el aprendizaje activo («learner-centered approach») (Lee, 2014; Freeman y otros, 2014). Es una metodología centrada en el proceso de aprendizaje, que fomenta la implicación del estudiante con la materia y con su propio proceso de aprendizaje.

Como aplicación de este concepto a las clases de prácticas del Grado en Comunicación Audiovisual, los estudiantes pueden aprender el manejo básico de las herramientas por medio de cursos en línea y utilizar las clases presenciales para dinámicas grupales.

La expresión audiovisual es una disciplina de alta complejidad cuyo aprendizaje no puede limitarse a las lecciones recibidas en un aula. La transferencia al estudiante de la responsabilidad de preparar las clases beneficia su desarrollo personal, la retención del conocimiento y la asimilación de los contenidos (Nygaard y Holtham, 2008; Wolff y otros, 2015). Schell y Mazur (2015) señalan que las personas aprenden mejor cuando están comprometidas, por lo que la clase invertida abre un camino sostenible de aprendizaje que alterna el trabajo previo a las clases, en el aula, y actividades después las clases. El esfuerzo previo del estudiante para conseguir dominar las herramientas de producción se puede utilizar como una base sobre la que estructurar un aprendizaje más profundo. Mintz (2014) expone cómo el modelo «blended learning» ayuda a que el estudiante aporte curiosidad, experiencia, conocimiento e ingenio, así como que se implique con el aprendizaje activo. El papel del profesor, de acuerdo con este autor, consiste en proporcionar una base estructural para el conocimiento, ofrecer la oportunidad de la puesta en práctica y proporcionar una retroalimentación comprometida. El profesor Horner (2016), especializado en la docencia de la técnica fotográfica, subraya la importancia para el proceso de aprendizaje de las presentaciones que hacen los estudiantes en el aula de sus ejercicios.

Un ejemplo de herramienta de aprendizaje semi-presencial son los cursos SPOC («Small Private Online Course»). En contraposición con los MOOC («Massive Open 
Online Course»), orientados a la difusión masiva del conocimiento, los SPOC son cursos preparados para un grupo concreto de estudiantes como refuerzo para la formación de las competencias que se están adquiriendo en el aula. Algunos autores han planteado que el formato de los libros de texto en el futuro puede ser una combinación entre «e-books» y SPOC (Fox, 2014; Burge, Fox, Grossman, Roth y Warren, 2015).

La Universidad Carlos III de Madrid puso en marcha en el año 2012 una estrategia de implementación de SPOC dirigidos a los estudiantes de nuevo ingreso que se realizan durante el verano antes de iniciar el Grado. El objetivo de esta iniciativa es asegurar unos conocimientos homogéneos en los grupos de docencia y un instrumento de estudio y repaso para los estudiantes. Por el momento se han producido SPOC de Física, Matemáticas, Biología o Química (Universidad Carlos III de Madrid, 2017). Siguiendo este modelo, la formación en las tecnologías de la producción audiovisual se podría articular a través de cursos introductorios antes de iniciar el grado, o también, por medio de un conjunto de SPOC complementarios a las distintas asignaturas que piden a los estudiantes realizar ejercicios prácticos en formato audiovisual.

En este artículo se presentan los resultados de una investigación realizada en la Universidad Carlos III de Madrid con estudiantes del Grado en Comunicación Audiovisual, con el objetivo de recoger sus opiniones sobre la implantación de cursos tipo SPOC para la formación básica en herramientas de producción audiovisual, siguiendo la línea de otros estudios centrados en la percepción de los estudiantes sobre el uso de vídeos para el aprendizaje en línea y en el aula (Rose, 2009), sobre la aplicación de métodos «blended learning» en la educación superior (Braun, 2008; López-Pérez, Pérez-López y Rodríguez-Ariza, 2011; Holzweiss, Joyner, Fuller, Henderson y Young, 2014) o sobre la experiencia «flipped learning» en la formación universitaria. La aportación original de este trabajo es el enfoque empírico en una línea de investigación en la que abundan las aproximaciones teóricas (López, Vázquez y Román, 2015). Se han perseguido tres objetivos:

- Identificar qué valores de utilidad destacan los estudiantes en relación con la implementación de herramientas «blended learning» como complemento a las clases presenciales de prácticas audiovisuales.

- Delimitar qué competencias del Grado en Comunicación Audiovisual pueden ser desarrolladas mediante herramientas «blended learning» para favorecer tanto la asimilación de conocimientos como el funcionamiento de las clases presenciales, según los estudiantes.

- Jerarquizar las propuestas obtenidas (valores de utilidad y competencias) en función de la importancia que los estudiantes les otorgan, con el fin de observar qué tipo de SPOC sería prioritario desarrollar. 


\title{
MATERIAL Y MÉTODOS
}

La investigación llevada a cabo se realizó de forma simultánea a la impartición de un curso SPOC piloto sobre edición de vídeo. Durante el mismo, se recopilaron y analizaron las opiniones de los estudiantes sobre la posible utilidad de los SPOC aplicados a la formación en producción audiovisual. Este análisis se llevó a cabo, inicialmente, mediante sesiones grupales de discusión («brainstorm» $\mathrm{y}$ «focus group») orientadas a partir de una serie de preguntas generales (figura 1) y, posteriormente, a través de encuestas individuales basadas en los resultados de los grupos de discusión.

Figura 1. Guía de preguntas iniciales para sesiones grupales

\author{
Guia para los grupos de discusión (Semana 1) \\ ¿Qué os parece el formato SPOC como complemento de las clases presenciales? \\ El área de especialización docente de los profesores que hemos lanzado esta \\ iniciativa es la formación en habilidades de operación de las tecnologías de la \\ producción audiovisual. ¿Qué necesidades de formación identificáis? \\ ¿Qué contenidos pensáis que podríamos desarrollar como SPOC de formación \\ complementaria al aula? \\ ¿Qué aspectos consideráis positivos o negativos en la utilización de este tipo de \\ cursos en vuestra carrera?
}

Como resultado de ese análisis, a partir de las respuestas a la pregunta «¿̨ué os parece el formato SPOC como complemento de las clases presenciales?», se obtuvieron distintos valores de utilidad o razones por las que un SPOC podría ser considerado como complemento para la enseñanza de materias relacionadas con la tecnología audiovisual.

Asimismo, de la pregunta «¿Qué tipo de contenidos de la titulación creéis que son susceptibles de tratarse mediante este tipo de metodologías en línea?», se delimitaron las competencias para las que un SPOC sería útil en este ámbito, según los participantes.

En ambos casos, los valores y competencias fueron categorizados (véase tabla 2 y tabla 3) y posteriormente jerarquizados por orden de prioridad a partir de los resultados obtenidos en una encuesta individual que recopilaba el listado completo con las sugerencias de la primera sesión (véase tabla 4 y tabla 5), con el fin de obtener pautas sobre cuáles podrían ser los contenidos de futuros SPOC. También se recogieron comentarios que ayudaran a contextualizar los resultados obtenidos. 
A continuación, se describe el SPOC piloto, así como las diferentes fases de la investigación y las herramientas empleadas para el análisis.

\section{Producción de un prototipo de SPOC para la investigación}

La investigación parte de un caso concreto: la producción, como proyecto piloto, de un SPOC cuyo objetivo docente es dotar a los estudiantes de las competencias básicas para poder realizar el montaje de un vídeo con el programa de edición DaVinci Resolve. Es una producción realizada por los profesores de Tecnología y Postproducción coordinada por Francisco Utray, en el formato corporativo que se utiliza en la universidad.

Se trata de un curso de tres semanas de duración con una carga de trabajo estimada para los estudiantes de cuatro horas semanales. DaVinci Resolve es un programa profesional de edición de vídeo y corrección de color que se distribuye de forma gratuita, y cuyo uso todavía no está muy extendido en la universidad. Al tratarse de un «software» libre, el SPOC se puede seguir tanto en las aulas informáticas de los laboratorios audiovisuales como con los equipos propios de los estudiantes.

Está previsto utilizar este SPOC al inicio del curso 2017/2018 para preparar a los estudiantes de nuevo ingreso a enfrentarse a la realización de una práctica sencilla de montaje de vídeo. El curso muestra las instalaciones disponibles en la universidad y las opciones de configuración del programa; aporta plantillas para trabajar la idea y el guion; hace recomendaciones sobre la organización de trabajo de montaje y los modos de edición; y propone un formato de exportación para la publicación de vídeos en Internet.

El curso piloto impartido expresamente para esta investigación se realizó entre el 27 de marzo y el 7 de abril de 2017 con una muestra de 22 estudiantes de distintos cursos del Grado de Comunicación Audiovisual. Se mezclaron estudiantes de los primeros cursos con otros ya más veteranos que pudieran expresar las dificultades con las que se habían encontrado para la realización de las prácticas a lo largo de sus estudios.

El curso consta de una serie de vídeos explicativos de una duración inferior a 6 minutos; ejercicios después de cada visionado para reforzar la asimilación de conceptos; y unas prácticas edición de vídeo.

A continuación, se desglosa el itinerario completo del curso:

Tabla 1. Itinerario del SPOC Edición de vídeo con DaVinci Resolve

\begin{tabular}{|c|l|}
\hline Semana & \multicolumn{1}{|c|}{ Contenido } \\
\hline o & Presentación del curso. \\
\hline 1 & $\begin{array}{l}\text { Creación de un proyecto, edición y exportación; Abrir el programa en un } \\
\text { ordenador de la universidad; La interfaz de usuario; Configuración de un } \\
\text { proyecto; Empezar a editar. }\end{array}$ \\
\hline
\end{tabular}




\begin{tabular}{|c|l|}
\hline Semana & \multicolumn{1}{|c|}{ Contenido } \\
\hline 2 & $\begin{array}{l}\text { Preparación del montaje y modos de edición; El guion de montaje; Visionado y } \\
\text { etiquetado del material; Edición de audio; Edición de vídeo; Rótulos y efectos. }\end{array}$ \\
\hline 3 & $\begin{array}{l}\text { Práctica libre; Transcodificación del material de cámara; Resumen del curso y } \\
\text { despedida. }\end{array}$ \\
\hline
\end{tabular}

\section{Desarrollo de la investigación}

La investigación se ha realizado mediante la combinación de herramientas cualitativas y cuantitativas, que han permitido obtener indicadores concretos e información complementaria para justificar y comprender los resultados obtenidos.

Para la obtención de datos con los que abordar los dos objetivos de la investigación, se ha desarrollado una estrategia metodológica que comprende las siguientes fases:

- Fase 1. Obtención de propuestas de los estudiantes. Se organizaron grupos de discusión con los estudiantes durante el desarrollo del curso en sucesivas etapas.

- Fase 2. Categorización. Incluyó un tratamiento cualitativo, con el fin de normalizar las respuestas en forma de posibles categorías. Asimismo, se añadió un tratamiento cuantitativo, con el fin de aplicar sobre los resultados una serie de posibles indicadores que permitieran la comparación de los resultados entre sí y su aplicación futura.

- Fase 3. Análisis. Creación de los indicadores de valores de utilidad y competencias desarrollables en cursos SPOC; y análisis de las justificaciones expresadas por los estudiantes en los grupos de discusión.

La figura 2 desglosa las tareas llevadas a cabo en cada una de las fases.

Figura 2. Desglose del desarrollo de la investigación: actividades realizadas por el equipo de investigación en letra negrita / actividades realizadas por los estudiantes en letra redonda

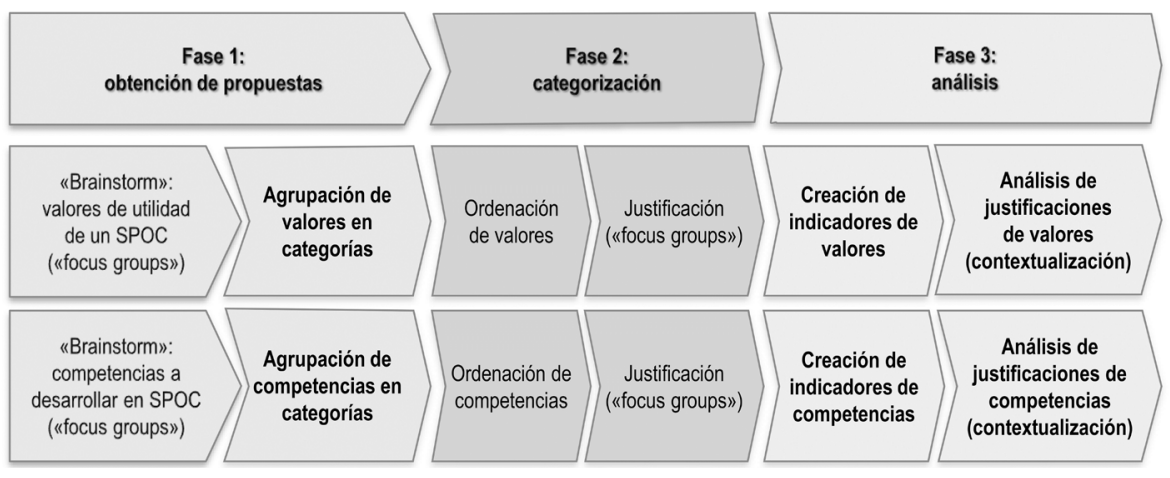




\section{Fase 1. Obtención de propuestas de los estudiantes}

El objetivo de la primera fase fue la obtención de propuestas por parte de los estudiantes, relacionadas con los dos objetivos de la investigación: 1) valores de utilidad de un SPOC y 2) competencias a desarrollar en formato SPOC.

Se dividió a los participantes para la realización de grupos de discusión o «focus groups» de 4 a 5 personas (Valles, 1999: p. 288). Cada sesión fue moderada por un profesor del equipo de investigación. Todas las conversaciones fueron grabadas y transcritas para su posterior análisis.

En cada grupo, se organizó una tormenta de ideas o «brainstorm» en torno a dos preguntas abiertas (Valles, 1999: p. 287). La primera de ellas, «¿Qué os parece el formato SPOC como complemento de las clases presenciales?», dio lugar a una conversación en donde los estudiantes se expresaron de forma espontánea sobre la experiencia que estaban realizando y las ventajas que este tipo de cursos les podría aportar. La segunda, «¿Qué tipo de contenidos de la titulación crees que son susceptibles de tratarse mediante este tipo de metodologías en línea?», tenía como objetivo que los estudiantes, en debate con sus propios compañeros, ofrecieran unos primeros indicios sobre el tipo de contenidos que les gustaría se desarrollaran en formato SPOC.

\section{Fase 2. Categorización}

El objetivo de la segunda fase fue organizar las respuestas, tanto relativas a los valores de utilidad de un SPOC como a las posibles competencias que se podrían desarrollar a través de dicho formato. Esta organización permitió agrupar las propuestas que se repetían y crear categorías comunes para cada objetivo que pudieran ser luego ordenadas de forma jerárquica, de mayor a menor utilidad por parte de los propios estudiantes.

Para ello, se crearon dos listas (de valores y de competencias), que fueron presentadas a los estudiantes en una nueva sesión de «focus group», durante la segunda semana del SPOC (tablas 2 y 3). No es posible recoger en este texto todas las respuestas obtenidas; no obstante, a continuación, se ejemplifican algunas con su correspondiente categorización: 
Tabla 2. Lista de respuestas obtenidas en la sesión «brainstorm» y su categorización

\begin{tabular}{|l|l|}
\hline \multicolumn{1}{|c|}{$\begin{array}{c}\text { Respuestas a «¿Qué os parece el } \\
\text { formato SPOC como complemento de presenciales?» }\end{array}$} & \multicolumn{1}{c|}{ Categorias (valores de utilidad) } \\
\hline $\begin{array}{l}\text { «El profesor podría ahorrar tiempo en } \\
\text { explicar cosas elementales» / «Estuvimos } \\
\text { una hora en para aprenderlo, y ahora } \\
\text { con el vídeo en dos minutitos queda todo } \\
\text { explicado» /... }\end{array}$ & $\begin{array}{l}\text { Evita las pérdidas de tiempo en las clases } \\
\text { presenciales para contenidos técnicos } \\
\text { básicos. }\end{array}$ \\
\hline $\begin{array}{l}\text { «A través de un SPOC puedes avanzar a tu } \\
\text { ritmo / «A veces somos muchos en clase y } \\
\text { aprendemos a velocidades distintas» / ... }\end{array}$ & $\begin{array}{l}\text { El alumno puede avanzar a su ritmo, repetir } \\
\text { el visionado de la lección tantas veces } \\
\text { como quiera o ir directo al contenido que le } \\
\text { interesa. }\end{array}$ \\
\hline $\begin{array}{l}\text { «En primer curso, no hacemos nada de esto } \\
\text { y para complementar nos viene muy bien» } \\
\text { / ... }\end{array}$ & $\begin{array}{l}\text { Es útil para adquirir una base en los } \\
\text { primeros cursos sobre una competencia } \\
\text { básica (edición de vídeo) que no se enseña } \\
\text { hasta cursos posteriores. }\end{array}$ \\
\hline $\begin{array}{l}\text { «Si hay un estudiante que ya conoce y otro } \\
\text { que no, el que no sabe puede hacer el SPOC } \\
\text { para adquirir más nivel» / ... }\end{array}$ & $\begin{array}{l}\text { Ayuda a los estudiantes que no conocen las } \\
\text { herramientas técnicas a adquirir un nivel } \\
\text { mínimo cercano al de compañeros que ya las } \\
\text { conocían. }\end{array}$ \\
\hline $\begin{array}{l}\text { «Estamos ya acostumbrados a aprender con } \\
\text { tutoriales» / ... }\end{array}$ & $\begin{array}{l}\text { Los estudiantes están acostumbrados } \\
\text { a aprender con tutoriales, por lo que es } \\
\text { positivo que las asignaturas se asemejen a } \\
\text { ese formato. }\end{array}$ \\
\hline $\begin{array}{l}\text { «Preferimos el formato visual / «Es mucho } \\
\text { más cómodo que el texto escrito» /... }\end{array}$ & $\begin{array}{l}\text { El formato visual de la explicación en vídeo } \\
\text { motiva más que el formato tradicional de } \\
\text { lecturas o guías. }\end{array}$ \\
\hline $\begin{array}{l}\text { «Sería bueno dedicarlo a contenidos más } \\
\text { /... }\end{array}$ & $\begin{array}{l}\text { Es un buen complemento para profundizar } \\
\text { en aspectos más avanzados. }\end{array}$ \\
\hline
\end{tabular}

Tabla 3. Lista de respuestas obtenidas en la sesión «brainstorm» y su categorización
Respuestas a «¿Qué tipo de contenidos
creéis que son susceptibles de tratarse
mediante este tipo de metodologías en
Categorías (competencias)

$$
\text { línea?» }
$$

«After Effects», «animación de efectos», «edición de color», «DaVinci», «rotoscopia» $/ \ldots$

Postproducción de vídeo: corrección de color / animación y efectos / rotoscopia. 


\begin{tabular}{|l|l|}
\hline $\begin{array}{c}\text { Respuestas a «¿Qué tipo de contenidos } \\
\text { creéis que son susceptibles de tratarse } \\
\text { mediante este tipo de metodologías en } \\
\text { línea?» }\end{array}$ & \multicolumn{1}{|c|}{ Categorias (competencias) } \\
\hline $\begin{array}{l}\text { «Cómo grabar sonido», «edición de audio», } \\
\text { «Audition» / .. }\end{array}$ & $\begin{array}{l}\text { Sonido: técnicas de grabación de sonido } \\
\text { directo y edición de audio. }\end{array}$ \\
\hline «Editar vídeos con Premiere»... & Edición de video. \\
\hline $\begin{array}{l}\text { «Conocimientos introductorios a cómo usar } \\
\text { los focos de la Universidad para iluminar», } \\
\text { «cómo se utilizan las cámaras» / ... }\end{array}$ & $\begin{array}{l}\text { Introducción básica a los equipos y } \\
\text { herramientas software de los laboratorios } \\
\text { del departamento: cámaras, grabadoras } \\
\text { de audio, Kit de iluminación, micrófonos, } \\
\text { equipos del plató de TV y del estudio de } \\
\text { radio, software de las aulas informáticas. }\end{array}$ \\
\hline «Photoshop», «Lightroom» / ... & $\begin{array}{l}\text { Fotografía digital: cámara DSLR y edición de } \\
\text { imagen fija. }\end{array}$ \\
\hline $\begin{array}{l}\text { «Indesign», «Illustrator» / ... } \\
\text { «iseño gráfico }\end{array}$ \\
\hline $\begin{array}{l}\text { «Consejos sobre cómo grabar una entrevista, poner un micro, etc.» / «Realización: } \\
\text { mesa de sonido, meter videos, pausar, } \\
\text { mezclar» / ... }\end{array}$ & $\begin{array}{l}\text { Realización: cómo grabar una entrevista y } \\
\text { otras técnicas (plano máster-insertos, plano- } \\
\text { contraplano, multicámara, etc.). }\end{array}$ \\
\hline «3D» / ... & Programas 3D para cine y TV. \\
\hline
\end{tabular}

Tras la categorización, en una segunda sesión se volvieron a organizar los grupos de discusión. En ellos se presentaron las listas para que, de forma individual, los participantes jerarquizaran cada opción en orden de mayor a menor importancia, dando lugar a los resultados que se presentan en las tablas 4 y 5 (Véase resultados).

Los valores de utilidad de un SPOC (por ejemplo, «para avanzar a mi ritmo») fueron jerarquizados en un rango desde 1 (opción con menor importancia) hasta 7 (opción con mayor importancia).

Por su parte, las propuestas de competencias a adquirir a través de cursos SPOC (por ejemplo, «para aprender postproducción de vídeo») fueron jerarquizadas en un rango desde 1 (opción con menor importancia) a 8 (opción con mayor importancia).

A continuación, se comentaron de forma pública las respuestas para que las justificaran, ayudados por un moderador, siguiendo un modelo similar al de la fase anterior. Con ello, se consiguió contrastar las opiniones de cada estudiante con las de sus compañeros y añadir un contexto a las justificaciones.

\section{Fase 3. Análisis de resultados}

Finalmente, se analizaron los resultados cuantitativos derivados de toda la investigación y las justificaciones expresadas en los grupos de discusión. 
Para el análisis de los datos se utilizaron varios indicadores, aplicados tanto a los valores de utilidad como a las posibles competencias a desarrollar en futuros SPOC. Estos indicadores son:

- Puntuación media de preferencia para los valores de utilidad $(\min =1 ; \max =7)$.

- Puntuación media de preferencia para las competencias $(\min =1 ; \max =8)$.

- Indicadores de desviación típica (dt): calculados por separado, tanto para los valores de utilidad como para las competencias. Muestran si hay respuestas que se alejan de la media, y cómo lo hacen. A menor desviación, más fiable es la puntuación media de preferencia.

- Indicadores de primera y última opción: indicadores secundarios sobre casos extremos, señalan el porcentaje de participantes que eligieron un determinado valor de utilidad o una competencia concreta como primera o última opción. En el caso de la primera opción, un porcentaje alto significaría que muchos estudiantes considerarían muy importante un valor de utilidad o una competencia. En el caso de la última opción, un porcentaje alto indicaría que no consideran prioritaria esa opción.

\section{RESULTADOS}

\section{Valores de utilidad de los SPOC comoherramienta complementaria a la formación presencial}

El primer objetivo de la investigación era identificar los valores por los que los estudiantes perciben la utilidad de los SPOC como complemento a las clases presenciales prácticas de producción audiovisual. A continuación, se muestran estos valores ordenados de mayor a menor preferencia media, acompañados de sus indicadores de desviación y elección como primera y última opción (tabla 4).

Tabla 4. Valores de utilidad sobre el uso de un SPOC como complemento a las clases presenciales de prácticas audiovisuales, según los estudiantes

\begin{tabular}{|l|c|c|c|c|}
\hline \multicolumn{1}{|c|}{ Valores de utilidad } & $\begin{array}{c}\text { Puntuación } \\
\text { media } \\
(1=\text { poco } \\
\text { preferente, } 7= \\
\text { muy preferente) }\end{array}$ & dt & $\begin{array}{c}\text { Primera } \\
\text { opción } \\
(\%)\end{array}$ & $\begin{array}{c}\text { Última } \\
\text { opción } \\
(\%)\end{array}$ \\
\hline $\begin{array}{l}\text { El alumno puede avanzar a su ritmo, repetir el } \\
\text { visionado de la lección tantas veces como quiera } \\
\text { o ir directo al contenido que le interesa. }\end{array}$ & 6,3 & 1,9 & $36 \%$ & $7 \%$ \\
\hline $\begin{array}{l}\text { Evita las pérdidas de tiempo en las clases } \\
\text { presenciales para contenidos técnicos básicos. }\end{array}$ & 5,6 & 2,3 & $29 \%$ & $21 \%$ \\
\hline
\end{tabular}




\begin{tabular}{|l|c|c|c|c|}
\hline \multicolumn{1}{|c|}{ Valores de utilidad } & $\begin{array}{c}\text { Puntuación } \\
\text { media } \\
(1=\text { poco } \\
\text { preferente, } 7= \\
\text { muy preferente) }\end{array}$ & dt & $\begin{array}{c}\text { Primera } \\
\text { opción } \\
(\%)\end{array}$ & $\begin{array}{c}\text { Última } \\
\text { opción } \\
(\%)\end{array}$ \\
\hline $\begin{array}{l}\text { Es útil para adquirir una base en los primeros } \\
\text { cursos sobre una competencia básica (edición de } \\
\text { vídeo) que no se enseña hasta cursos posteriores. }\end{array}$ & 5,6 & 2,0 & $21 \%$ & $0 \%$ \\
\hline $\begin{array}{l}\text { El formato visual de la explicación en vídeo } \\
\text { motiva más que el formato tradicional de } \\
\text { lecturas o guías. }\end{array}$ & 5,4 & 1,9 & $7 \%$ & $14 \%$ \\
\hline $\begin{array}{l}\text { Ayuda a los estudiantes que no conocen las } \\
\text { herramientas técnicas a adquirir un nivel } \\
\text { mínimo cercano al de compañeros que ya las } \\
\text { conocían. }\end{array}$ & 4,5 & 1,4 & $7 \%$ & $0 \%$ \\
\hline $\begin{array}{l}\text { Ya estamos acostumbrados a aprender con } \\
\text { tutoriales, por lo que es positivo que las } \\
\text { asignaturas se asemejen a ese formato. }\end{array}$ & 4,2 & 1,6 & $0 \%$ & $21 \%$ \\
\hline $\begin{array}{l}\text { Es un buen complemento para profundizar en } \\
\text { aspectos más avanzados. }\end{array}$ & 3,4 & 1,6 & $0 \%$ & $36 \%$ \\
\hline
\end{tabular}

Se observa que la opción «El alumno puede avanzar a su ritmo, repetir el visionado de la lección tantas veces como quiera o ir directo al contenido que le interesa» es el valor de utilidad más importante para los participantes, con una puntuación media de 6,3 sobre 7 puntos y una desviación típica relativamente baja $(1,9)$ que indica que esta es una opinión compartida por buena parte del grupo. Un tercio de los estudiantes la coloca como la opción más valorada, y muy pocos la eligen como última opción (7\%). Durante las entrevistas abiertas los estudiantes justificaron esta opción con las siguientes afirmaciones:

- «Permite personalizar el aprendizaje, adaptarlo a cada estudiante y a los tiempos que necesita para asimilar».

- «Puedes apuntar las dudas que te surgen en casa tras ver el vídeo y luego en la clase presencial el profesor te las puede responder».

El segundo valor priorizado por los estudiantes pone el foco en la complementariedad del SPOC con la clase presencial: «Evita las pérdidas de tiempo en las clases presenciales para contenidos técnicos básicos». Casi un tercio de los participantes lo señalaron como primera opción, pero el 21\% como última opción. Por lo tanto, existe una gran discrepancia en este punto $(\mathrm{dt}=2,3)$. Los estudiantes argumentaron esta opción desde un punto de vista pragmático: 
- «Es muy útil para el profesor, porque se ahorra el tiempo de explicar cosas que son elementales, y para el alumno, que puede adelantar en casa y aprovechar mejor la clase presencial».

- «Lleva menos tiempo que las clases para aprender lo mismo».

«Es útil para adquirir una base en los primeros cursos sobre una competencia básica (edición de vídeo) que no se enseña hasta cursos posteriores» tiene una puntuación media alta $(5,6)$, y es una opinión compartida por buena parte de los estudiantes $(\mathrm{dt}=2,0)$. Ninguno de los participantes la considera como última opción. En las discusiones abiertas los estudiantes remarcaron la conveniencia de que estos materiales didácticos estuvieran disponibles para consulta y repaso.

- «(...) si lo tienes en un vídeo y lo puedes volver a ver, es muy útil».

- «Sería bueno que el SPOC estuviera siempre abierto para consulta durante toda la carrera».

Por su parte, la idea de que «El formato visual de la explicación en vídeo motiva más que el formato tradicional de lecturas o guías» también está considerada entre las primeras opciones $(5,4)$ y tiene un nivel de desviación bajo $(\mathrm{dt}=1,9)$. Asimismo, son bajos los porcentajes de elección como primera y última opción indicando que es considerado como un buen valor de utilidad, aunque no prioritario.

La opción «Ayuda a los estudiantes que no conocen las herramientas técnicas a adquirir un nivel mínimo cercano al de compañeros que ya las conocían» es una opción menos priorizada con una puntuación media $(4,5)$ y una desviación pequeña $(\mathrm{dt}=1,4)$. Ninguno de los participantes la eligió como última opción. La argumentación de este valor también se puso en relación con la eficacia de las dinámicas docentes de la clase presencial:

- «Gracias a estos vídeos, los estudiantes rezagados se pueden poner al nivel del resto de la clase. De esta forma el profesor no tiene que ir uno por uno explicando cómo se hace cada cosa».

Por último, los estudiantes no han respaldado mayoritariamente la opción «... estamos acostumbrados a aprender con tutoriales...», basándose en su presunta costumbre con este tipo de recursos. Tampoco han priorizado los SPOC como herramienta para cursos avanzados. En ambos casos coinciden puntuaciones medias bajas, con desviaciones típicas también bajas y un porcentaje significativo de estudiantes ponen alguna de estas opciones como última opción. 


\section{Competencias sugeridas por los estudiantes a desarrollar mediante SPOC}

En cuanto a las propuestas de cursos SPOC enfocados a competencias específicas de operación de las tecnologías de la producción audiovisual, se presenta a continuación una lista ordenada según su preferencia media, así como la desviación típica de dicha puntuación y los porcentajes de elección de cada elemento como primera y última opción:

Tabla 5. Lista de competencias sugeridas por los estudiantes para posibles futuros SPOC (dt máxima $=3,6$ )

\begin{tabular}{|l|l|l|l|l|}
\hline \multicolumn{1}{|c|}{ Competencias a desarrollar en formato SPOC } & $\begin{array}{c}\text { Puntuación } \\
\text { media } \\
(1=\text { poco } \\
\text { preferente, } 8= \\
\text { muy preferente) }\end{array}$ & D.T. & $\begin{array}{c}\text { Primera } \\
\text { opción } \\
(\%)\end{array}$ & $\begin{array}{l}\text { Última } \\
\text { opción } \\
(\%)\end{array}$ \\
\hline $\begin{array}{l}\text { Postproducción de vídeo: corrección de color / } \\
\text { animación y efectos / rotoscopia. }\end{array}$ & 5,9 & 1,7 & $14 \%$ & $0 \%$ \\
\hline $\begin{array}{l}\text { Sonido: técnicas de grabación de sonido directo } \\
\text { y edición de audio. }\end{array}$ & 5,6 & 1,8 & $14 \%$ & $0 \%$ \\
\hline Edición de video. & 5,4 & 2,6 & $14 \%$ & $38 \%$ \\
\hline $\begin{array}{l}\text { Introducción básica a los equipos y } \\
\text { herramientas software de los laboratorios del } \\
\text { departamento: cámaras, grabadoras de audio, } \\
\text { Kit de iluminación, micrófonos, equipos del } \\
\text { plató de TV y del estudio de radio, software de } \\
\text { las aulas informáticas. }\end{array}$ & 4,9 & 2,2 & $29 \%$ & $0 \%$ \\
\hline $\begin{array}{l}\text { Fotografía digital: cámara DSLR y edición de } \\
\text { imagen fija. }\end{array}$ & 4,9 & 2 & $14 \%$ & $0 \%$ \\
\hline Diseño gráfico. & 3,6 & 2,2 & $7 \%$ & $25 \%$ \\
\hline $\begin{array}{l}\text { Realización: cómo grabar una entrevista y } \\
\text { otras técnicas (plano máster-insertos, plano- } \\
\text { contraplano, multicámara etc.). }\end{array}$ & 3,6 & 2,3 & $7 \%$ & $25 \%$ \\
\hline Programas 3D para cine y TV. & 1,7 & $0 \%$ & $88 \%$ \\
\hline
\end{tabular}


Según las puntuaciones medias que recibió cada competencia, las habilidades relacionadas con la postproducción de vídeo $(5,9)$, la captación y el tratamiento digital del sonido $(5,6)$ y la edición de vídeo $(5,6)$ son las mejor valoradas para cursos en formato SPOC. Se puede considerar que las apreciaciones sobre postproducción y sonido cuentan con cierto consenso en el grupo al presentar las desviaciones típicas más bajas $(\mathrm{dt}=1,7$ y 1,8), mientras que la opción de edición de vídeo presenta una mayor discrepancia $(\mathrm{dt}=2,6) \mathrm{y}$, además, un relativamente alto porcentaje de estudiantes (38\%) la considera como la competencia menos prioritaria.

La vocación homogénea por la postproducción de vídeo y audio se tiene que matizar teniendo en consideración que la muestra está compuesta por voluntarios que han elegido participar en un curso piloto de DaVinci, un programa muy atractivo para estudiantes con afición por la postproducción y la corrección de color. Por este motivo, no se considera un resultado concluyente y se estima necesaria una investigación con una muestra más amplia de estudiantes que puedan tener otros intereses prioritarios. También se necesitan más datos para justificar el alto porcentaje de estudiantes que sitúa la edición de vídeo como última opción.

En cuanto a la introducción básica a los equipos y el «software» de los laboratorios, si bien no es considerada tan prioritaria por todos los estudiantes (media $=4,9$; $\mathrm{dt}$ $=2,2$ ), sí es destacable que un gran número de ellos la consideran como primera opción (29\%, el valor más alto de todas las opciones) y que nadie la considera como última opción. La interpretación de estos datos indica que es un contenido que goza de un respaldo amplio por un conjunto significativo de los participantes.

La competencia relativa a la fotografía digital tiene valores equivalentes, aunque un poco inferiores, a la edición de vídeo, audio y postproducción.

Las competencias puntuadas como menos urgentes para su desarrollo mediante cursos SPOC fueron las técnicas de realización (media $=3,6$ ), el diseño gráfico (media $=3,6) \mathrm{y}$, especialmente, las relacionadas con el manejo de herramientas $3 \mathrm{D}$ para cine $\mathrm{y}$ televisión (media $=2,3$ ). Las desviaciones típicas de estas tres opciones $(\mathrm{dt}=2,2$; 2,1 y 1,7 respectivamente) denotan cierto consenso en no priorizar el aprendizaje de las técnicas $3 \mathrm{D}$ y, sin embargo, mayor discrepancia para las respuestas sobre realización y el diseño gráfico.

En los grupos de discusión, los estudiantes justificaron la baja prioridad que le otorgaban a la creación de un SPOC sobre los sistemas de grafismo 3D (un 88\% de los estudiantes señalaron esta opción como la última en sus preferencias) con los siguientes argumentos:

- «...(3D) es demasiado complejo».

- «... no me parece tan importante porque lo que nos van a pedir en unas prácticas en un trabajo es edición de vídeo y de sonido». 


\section{DISCUSIÓN Y CONCLUSIONES}

En relación con la hipótesis de partida -las metodologías docentes que combinan el aprendizaje en línea con el presencial en el Grado en Comunicación Audiovisual favorecen la adquisición de competencias básicas de operación de los dispositivos tecnológicos de la producción audiovisual-, esta se ve tendencialmente confirmada por los resultados obtenidos tras la experiencia del grupo participante con el SPOC de edición de vídeo y los posteriores diálogos sobre su utilidad y sus posibles aplicaciones.

En concreto, los resultados de la investigación han permitido identificar con claridad algunas tendencias actitudinales de los estudiantes ante estos nuevos formatos e iniciar una reflexión sobre modelos educativos más eficaces para formación en tecnologías para la producción audiovisual.

Cabe destacar, en primer lugar, que los participantes valoran positivamente los soportes multimedia interactivos para el autoaprendizaje y su uso complementario con la enseñanza presencial.

Los objetivos de identificar y jerarquizar los valores de utilidad, que destacan los estudiantes en los SPOC, y las competencias del Grado en Comunicación Audiovisual, que son susceptibles de enseñarse con estos formatos, han encontrado una respuesta concreta: un porcentaje significativo dentro del grupo se ha decantado por la opción «introducción básica a los equipos y herramientas software de los laboratorios del departamento», mediante unos cursos que estén abiertos y accesibles para «avanzar a su propio ritmo y repetir el visionado de la lección tantas veces como quiera». De la combinación de estas dos respuestas, se puede extraer que los participantes apoyan mayoritariamente la producción de un SPOC específico que, por una parte, les ayudara en una etapa inicial de formación básica en el manejo de los dispositivos que van a seguir utilizando a lo largo de la carrera y que estos conocimientos, por otra, puedan ser asimilados por cada estudiante en el tiempo que este necesite, así como revisitados en cursos posteriores.

En este sentido, es destacable que durante la fase de «brainstorming» todos los participantes reconocieron carencias de formación técnica en habilidades de operación de la tecnología de producción audiovisual. En los grupos de discusión, este tema se relacionaba habitualmente con la ventaja que supondría implementar el SPOC para cuestiones iniciales básicas.

En relación con la clase presencial, los estudiantes valoraron como importante que «[el SPOC] evita las pérdidas de tiempo en las clases presenciales para contenidos técnicos básicos», de lo que se concluye que los soportes de formación en línea contribuyen a que la clase en el aula sea más eficaz y que pueda orientarse hacia la profundización de contenidos, relegando los más básicos a los cursos SPOC.

También han sido frecuentes las intervenciones en las que los participantes consideran que este formato de enseñanza ayudaría a los estudiantes que carecen de preparación previa y que podrían «adquirir un nivel mínimo cercano al de compañeros 
que ya conocían las herramientas técnicas». Este aspecto resulta especialmente relevante ya que conecta con el problema identificado por los profesores en las aulas y que constituía el punto de partida para este artículo: la disparidad de niveles de operación técnica de los estudiantes que forman los grupos de docencia y cómo esta podría solventarse mediante herramientas de aprendizaje «blended learning».

No obstante, las percepciones sobre sus propias carencias formativas son distintas. Unos piensan que estas son más críticas en el ámbito del sonido; otros que necesitan más formación en fotografía; y muchos demandan más formación y más temprana para la postproducción de vídeo.

Cabe destacar que en el desarrollo de los grupos de discusión ninguno de los estudiantes se ha mostrado negativo hacia el uso de los SPOC. Por lo tanto, se identifica una tendencia homogénea hacia la consideración de los recursos didácticos en línea como un complemento útil para la formación en habilidades de operación de equipos de los laboratorios audiovisuales. No obstante, la muestra cuenta con el sesgo de que todos los estudiantes han participado voluntariamente en la realización del SPOC, por lo que su opinión inicial sobre esta herramienta podría ser positiva de antemano, y la realización del curso no les habría hecho cambiar de opinión.

La muestra de estudiantes con la que se ha hecho esta investigación es demasiado pequeña para el análisis estadístico y no se pueden obtener a partir de ella resultados cuantitativos concluyentes. Pero sí se han observado tendencias que son una valiosa fuente para definir una futura investigación con el mismo objeto de estudio. Ese proyecto de investigación podrá implicar a la totalidad de los estudiantes de nuevo ingreso en el Grado e incorporar una metodología de análisis de efectividad (MuñozMerino, Ruipérez-Valiente, Alario-Hoyos, Pérez-Sanagustín y Delgado Kloos, 2015).

\section{REFERENCIAS BIBLIOGRÁFICAS}

Alexander, S. (1999). An Evaluation of Innovative Projects Involving Communication and Information Technology in Higher Education. Higher Education Research \& Development, 18(2), 173-183.

ANECA. (2006). Libro Blanco. Títulos de Grado en Comunicación. Recuperado de https://goo.gl/CC699a

Bartolomé, A., y Aiello, M. (2006). Nuevas tecnologías y necesidades formativas. Blended Learning y nuevos perfiles en comunicación audiovisual. Telos. Cuadernos de Comunicación. Tecnologia y Sociedad(67).

Bartolomé, A., y Steffens, K. (2015). ¿Son los MOOC una alternativa de aprendizaje?
Comunicar, 44(XXII), 91-99. doi:10.3916/ C44-2015-10.

Bernard, R. M., Abrami, P. C., Lou, Y., Borokhovski, E., Wade, A., Wozney, L., y Huang, B. (2004). How Does Distance Education Compare With Classroom Instruction? A Meta-Analysis of the Empirical Literature. Review of Educational Research, 74(3), 379-439. doi: 10.3102/00346543074003379.

Braun, T. (2008). Making a Choice: The Perceptions and Attitudes of Online Graduate Students. Journal of Technology and Teacher Education, 16(1), 63-92.

Burge, J., Fox, A., Grossman, D., Roth, G., y Warren, J. (2015). SPOCs: What, Why, and How. Proceedings of the 46th ACM 
Technical Symposium on Computer Science Education (págs. 595-596). ACM. Chiappe-Laverde, A., Hine, N., y MartínezSilva, J. (2015). Literatura y práctica: una revisión crítica acerca de los MOOC. Comunicar, 44(XXII), 9-18. doi:10.3916/ C44-2015-01.

Fox, A. (2014). Curricular Technology Transfer for the 21st Century: MOOCs and technology to advance learning and learning research. Ubiquity, June. doi: $10.1145 / 2618397$.

Freeman, S., Eddy, S. L., McDonough, M., Smith, M. K., Okoroafor, N., Jordt, H., y Wenderoth, M. P. (2014). Active learning increases student performance in science, engineering, and mathematics. Proceedings of the National Academy of Sciences, 111(23), 8410-8415. doi: 10.1073/pnas.1319030111.

Graham, C. R. (2004). Blended learning systems: definition, current trends, and future directions. En C. J. Bonk, y C. R. (Eds.), The handbook of blended learning: Global perspectives, local designs (3-21). Zurich: Pfeiffer Publishing.

Harding, A., Kaczynski, D., y Wood, L. (2005). Evaluation of blended learning: analysis of qualitative data. Proceedings of uniserve science blended learning symposium, (56-61).

Holzweiss, P. C., Joyner, S. A., Fuller, M. B., Henderson, S., y Young, R. (2014). Online graduate students' perceptions of best learning experiences. Distance Education, 35(3), 311-323. doi: 10.1080/01587919.2015.955262.

Lee, C. P. (2014). The effects of interactive discourse, the Socratic method, and active learning labs on student achievement at the university level. A comparative approach. Texas A\&M University. Texas A\&M University.

Lim, D. H., y Kim, H. J. (2003). Motivation and learner characteristics affecting online learning and learning application.
Journal of Educational Technology Systems, 31(4), 423-439.

López, E., Vázquez, E., y Román, P. (2015). Análisis e implicaciones del impacto del movimiento MOOC en la comunidad científica: JCR y Scopus (2010-13). Comunicar, 44(XXII), 73-80. doi: 10.3916/C44-2015-08.

López-Pérez, M. V., Pérez-López, M. C., y Rodríguez-Ariza, L. (2011). Blended learning in higher education: Students' perceptions and their relation. Computers \& Education(56), 818-826. doi: 10.1016/j. compedu.2010.10.023.

Muñoz-Merino, P. J., Ruipérez-Valiente, J. A., Alario-Hoyos, C., Pérez-Sanagustínc, M., y Delgado Kloos, C. (2015). Precise Effectiveness Strategy for analyzing the effectiveness of students with educational resources and activities in MOOCs. Computers in Human Behavior, 47, 108118. doi: 10.1016/j.chb.2014.10.003.

Nunan, T., George, R., y McCausland, H. (2000). Rethinking the ways in which teaching and learning are supported: the flexible centre at the University of South Australia. Journal of Higher Education Policy and Management, 22(1), 85-98.

Nygaard, C., y Holtham, C. (2008). Understanding learning-centred higher education. Copenhagen Business School Press DK.

Porter, W. W., y Graham, C. R. (2016). Institutional drivers and barriers to faculty adoption of blended. British Journal of Educational Technology, 47(4), 748-762. doi: 10.1111/bjet.12269.

Rose, K. (2009). Student Perceptions of the Use of Instructor-Made Videos in Online and Face-to-Face Classes. MERLOT Journal of Online Learning and Teaching , 5(3).

Schell, J., y Mazur, E. (2015). Flipping the chemistry classroom with peer instruction. En J. García-Martínez, E. Seerano-Torregrosa, \& (Eds.), Chemistry 
Education: Best Practices, Opportunities and Trends. Willey Online Library.

Universidad Carlos III de Madrid (2017). Competencias que otorga el Grado en Comunicación Audiovisual. Recuperado de https://goo.gl/SpMLhF

Utray, F. (2016). Implantando la metodología "flipped classroom" en la asignatura 'Postproducción Digital' en la Universidad Carlos III de Madrid. En Sánchez Cid, M (coord.), Didácticas y modelos formativos en asignaturas de comunicación audiovisual, publicidad y periodismo. Dykinson.
Valles, M. S. (1999). Técnicas cualitativas de investigación social. Reflexión metodológica y práctica profesional. Madrid: Síntesis.

Voos, R. (2003). Blended learning. What is it and where might it take us? Sloan-C View, 2(1), 2-5.

Wolff, M., Wagner, M. J., Poznanski, S., Schiller, J., y Santen, S. (2015). Not another boring lecture: engaging learners with active learning techniques. The Journal of emergency medicine, 48(1), 85-93.

\section{PERFIL ACADÉMICO Y PROFESIONAL DE LOS AUTORES}

Ana Mejón. Personal Investigador en Formación (PIF) del Departamento de Periodismo y Comunicación Audiovisual de la Universidad Carlos III de Madrid. Miembro del grupo de investigación TECMERIN. Sus líneas de investigación giran en torno a la producción y las tecnologías audiovisuales.

E-mail: amejon@hum.uc3m.es

Francisco Utray. Francisco Utray Delgado es Profesor Titular de Comunicación Audiovisual en la Universidad Carlos III de Madrid (UC3M), miembro del grupo de investigación TECMERIN y asesor de producción audiovisual de la Unidad de Tecnología Educativa e Innovación Docente de la UC3M (UTEID). Sus líneas de investigación giran en torno a las tecnologías audiovisuales.

E-mail: futray@hum.uczm.es

David Rodríguez-Mateos. David Rodríguez Mateos es profesor en el Departamento de Periodismo y Comunicación Audiovisual de la Universidad Carlos III de Madrid. Miembro del grupo de investigación Tecnodoc. Docente en tecnología audiovisual y tecnologías de la información. Ha participado como gestor y profesor en cursos universitarios de grado y posgrado, impartidos mediante formación a distancia, desde 2007 hasta la actualidad. Su actual línea de investigación es el análisis de datos cuantitativos sobre la evolución de los alumnos y sus resultados en cursos a distancia (learning analytics).

E-mail: pirio@hum.uc3m.es 


\section{DIRECCIÓN DE LOS AUTORES}

Facultad de Humanidades, Comunicación y Documentación.

Universidad Carlos III de Madrid

C/ Madrid, 133

28903 Getafe (Madrid, España)

Fecha de recepción del artículo: 31/07/2017

Fecha de aceptación del artículo: 17/10/2017

\section{Como citar este artículo:}

Mejón, A., Utray, F., y Rodríguez Mateos, D. (2018). Opiniones y propuestas de los estudiantes de Comunicación Audiovisual sobre los SPOC. RIED. Revista Iberoamericana de Educación a Distancia, 21(2), pp. 305-324. doi: http://dx.doi. org/10.5944/ried.21.2.19363 